Ciência Florestal, Santa Maria, v. 21, n. 1, p. 1-8, jan.-mar., 2011

ISSN 0103-9954

\title{
ANÁLISE TEMPORAL DA VEGETAÇÃO NA REGIÃO DO NORDESTE ATRAVÉS DE DADOS EVI DO MODIS
}

\author{
MULTI-TEMPORAL ANALYSIS OF NORTHEAST VEGETATION BY MEANS \\ OF MODIS-EVI DATA
}

Mileide de Holanda Formigoni ${ }^{1}$ Alexandre Cândido Xavier ${ }^{2}$ Julião Soares de Souza Lima ${ }^{3}$

RESUMO

O Nordeste Brasileiro (NEB) apresenta diferentes tipos de vegetação, sendo importantes para manutenção do seu ecossistema. Com a utilização de técnicas de sensoriamento remoto é possível analisar variações de comunidades de vegetação e suas alterações fenológicas. O objetivo principal deste trabalho é avaliar o comportamento temporal do Índice de Vegetação Melhorado (EVI) do sensor Moderate Resolution Imaging Spectroradiometer (MODIS), de diferentes tipos de vegetação do NEB no período entre fevereiro de 2000 a julho de 2006. A área de estudo foi um transecto de comprimento aproximadado $1.800 \mathrm{~km}$ sob a latitude $-6^{\circ} 41^{\prime} 24^{\prime \prime}$ localizada na região do NEB, onde foi possível avaliar os principais tipos de vegetação existentes. Para a caracterização dos tipos de vegetação, foi utilizado um mapa de vegetação na escala de 1:5.000.000 do Instituto Brasileiro de Geografia e Estatística (IBGE). Para se cobrir o período estudado, foi utilizado um total de 144 imagens EVI livres de nuvens com resolução espacial de $250 \mathrm{~m}$, adquiridas da Agencia Nacional Aeroespacial Norte-americana (NASA). Os resultados obtidos mostraram que: i) os dados EVI foram sensíveis aos diferentes tipos de vegetação; ii) a vegetação Amazônia apresentou a menor variação multitemporal dos valores de EVI, todavia apresentando os valores mais elevados; iii) a vegetação de Caatinga analisada apresentou a maior variação dos valores de EVI.

Palavras-chave: sensoriamento remoto; índices de vegetação; biomas.

\begin{abstract}
The Brazilian Northeast (NEB) region presents different vegetation types that are important to maintain this ecosystem. With remote sensing techniques it is possible to analyze variations in vegetation community and alterations in vegetation phenology. The main objective of this work is to evaluate the temporal behavior of the Enhanced Vegetation Index (EVI) from Moderate Resolution Imaging Spectroradiometer (MODIS) of different vegetation types in the NEB over the period ranging from February/2000 to July/2006. The study area was a 1,800 km long transect at latitude $-6^{\circ} 41$ '24" enclosed at the NEB region. A map of Brazil (1:5,000,000 scale, from Brazilian Institute of Geography and Statistics, IBGE) was used to characterize the vegetation types. A total of 144 cloud-free EVI images with spatial resolution of $250 \mathrm{~m}$ were acquired from National Aeronautics and Space Administration (NASA). The results showed that: i) EVI data were sensible to the vegetation types; ii) amazon vegetation presented lesser variation in the multi-temporal EVI, however with greater values; iii) Caatinga vegetation presented greater EVI values variation.
\end{abstract}

Keywords: remote sensing; vegetation indices; biomes.

1. Bióloga, MSc., Alto Universitário, s/n, Caixa Postal 16, Guararema, CEP 29500-000, Alegre (ES). mileidehf@gmail.com

2. Engenheiro Agrícola, Dr., Professor Adjunto do Departamento de Engenharia Rural, Centro de Ciências Agrárias, Universidade Federal do Espírito Santo, Alto Universitário, s/n, Caixa Postal 16, Guararema, CEP 29500-000, Alegre (ES).xavier@cca.ufes.br

3. Engenheiro Agrícola, Dr., Professor Associado do Departamento de Engenharia Rural, Centro de Ciências Agrárias, Universidade Federal do Espírito Santo, Alto Universitário, s/n, Caixa Postal 16, Guararema, CEP 29500-000, Alegre (ES).limajss@yahoo.com.br

Recebido para publicação em 14/03/2008 e aceito em 31/08/2010.

Ci. Fl., v. 21, n. 1, jan.-mar., 2011 


\section{INTRODUÇÃO}

O Nordeste Brasileiro (NEB), localizado na faixa tropical de aproximadamente $1^{\circ}$ a $18^{\circ}$ de latitude Sul e $35^{\circ}$ a $47^{\circ}$ de longitude Oeste, é constituído pelos estados de Alagoas (AL), Bahia $(\mathrm{BA})$, Ceará (CE), Maranhão (MA), Paraíba (PB), Pernambuco (PE), Piauí (PI), Rio Grande do Norte $(\mathrm{RN})$ e Sergipe (SE). O NEB é uma região que apresenta alta diversidade biológica, onde podem ser encontrados diversos biomas e tipos de vegetação. Tendo como base o mapa de bioma do Brasil, na escala de 1:5.000.000, os principais biomas encontrados no NEB são: amazônia, caatinga, cerrado e mata atlântica (IBGE, 2004a).

O monitoramento da cobertura vegetal, utilizando produtos e técnicas de Sensoriamento Remoto (SR) e geoprocessamento é embasado na necessidade de análise dos recursos vegetais, contribuindo para o acompanhamento temporal e a obtenção de informações como a distribuição dos tipos de vegetação, a fenologia, a estrutura do dossel, as condições de estresse e as mudanças de uso do solo. Uma das maneiras de se realizar estudos relacionados à vegetação é por meio de técnicas de SR, utilizando Índices de Vegetação espectrais (IVs), que estão relacionados, dentre outros fatores, ao índice de área foliar (TUCKER et al., 1985).

$\mathrm{Na}$ literatura, são encontrados diversos IVs, sendo que a maioria deles é obtida através de medidas da reflectância nas faixas espectrais do vermelho ( $\rho_{V e r} \sim 0,62$ a $\left.0,67 \mu \mathrm{m}\right)$ e do infravermelho próximo $\left(\rho_{I V P} \sim 0,70\right.$ a $\left.0,90 \mu \mathrm{m}\right)$ do espectro eletromagnético (MOREIRA \& SHIMABUKURO, 2004). Pode-se citar como exemplo de IVs, o NDVI (Índice de Vegetação por Diferença Normalizada), o SAVI (Índice de Vegetação Ajustado ao Solo) e o EVI (Índice de Vegetação Melhorado).

O EVI foi desenvolvido para se trabalhar com dados do sensor MODIS, a bordo dos satélites Terra e Acqua, lançados pela NASA (Agência Nacional Aeroespacial Norte-americana (HUETE et al., 1997), sendo este IV mais sensível ao sinal da vegetação verde, minimizando os efeitos do solo e da atmosfera (JUSTICE et al., 1998), e calculado como:

$$
E V I=G \frac{\rho_{I V P}-\rho_{V e r}}{L+\rho_{I V P}+C_{1} \rho_{V e r}-C_{2} \rho_{a z u l}}
$$

em que: $\mathrm{G}=$ fator de ganho $(2,5) ; \rho_{I V P}$ $=$ reflectância no infravermelho próximo; $\rho_{V e r}=$ reflectância no vermelho; $\rho_{\text {Azul }}=$ reflectância no azul; $\mathrm{C}_{1}=$ coeficiente de correção dos efeitos atmosféricos para o vermelho (6); $\mathrm{C}_{2}=$ coeficiente de correção dos efeitos atmosféricos para o azul $(7,5) ; \mathrm{L}=$ fator de correção para a interferência do solo.

Vários estudos já foram realizados utilizando IVs. Ferreira et al. (2005) analisaram a sazonalidade do bioma cerrado através do NDVI e EVI do sensor MODIS. Comparando os IVs, observaram que o EVI apresentou um melhor desempenho para a discriminação sazonal da cobertura vegetal, por ser mais sensível a pequenas variações do IAF. Já Lacruz et al. (2005) realizaram o monitoramento das mudanças em um corredor ecológico do bioma amazônia no estado do Pará, no período de jan/2001 a dez/2003, por meio de imagens EVI derivadas do sensor MODIS com resolução espacial de 250 $\mathrm{m}$. Através de análise harmônica, caracterizaram o comportamento de diferentes tipologias de uso e cobertura da terra. As imagens EVI/MODIS se mostraram apropriadas para esse tipo de estudo, principalmente quando se requer trabalhar com extensas regiões.

Imagens multi-temporais de IVs têm se mostrado eficientes para o monitoramento da vegetação (Justice et al., 1985), uma vez que, utilizando apenas uma imagem, pode-se ter tipos de vegetação distintos, todavia com comportamento espectral semelhante (Townshend et al., 1987).

Este trabalho tem como objetivo principal avaliar o comportamento temporal do Índice de Vegetação Melhorado (EVI) do sensor MODIS para os principais biomas do NEB, inseridos no transecto de aproximadamente $1800 \mathrm{~km}$ (IBGE, 2004a) na latitude $-6^{\circ} 41^{\prime} 24^{\prime \prime}\left(-6,69^{\circ}\right)$, no período de fevereiro de 2000 a julho de 2006 .

\section{MATERIAIS E MÉTODOS}

A área de estudo foi a região Nordeste do Brasil, mais especificamente o transecto com comprimento de aproximadamente $1800 \mathrm{~km}$ (IBGE, 2004a), sob a latitude $-6,69^{\circ}$. Essa latitude foi escolhida por conter, ao longo das longitudes, os principais biomas do NEB: amazônia, caatinga, cerrado e mata atlântica. A Figura 1 ilustra o transecto da latitude $-6,69^{\circ}$, onde são identificados os principais tipos de vegetação inseridos nos principais biomas do NEB, a partir do mapa de vegetação do IBGE (2004b).

De acordo com a classificação de Köeppen, o clima do NEB é classificado como Bshw (quente 
e semi-árido, com chuvas de verão) e Bshw' (quente semi-árido, com chuvas de verão/outono), havendo a influência da massa equatorial continental, da massa equatorial atlântica e da massa tropical atlântica, portanto, todo esse sistema tem influência no ambiente (FERNANDES, 2003). Com relação à temperatura, o NEB apresenta certa homogeneidade espacial e pouca variação anual. No litoral nordestino, a temperatura média anual está em torno dos $24^{\circ} \mathrm{C}$. A mínima média do mês mais frio é de $26^{\circ} \mathrm{C}$ do $\mathrm{RN}$ ao SE e de $22^{\circ} \mathrm{C}$ na BA (SILVA, 1987).

Para definição e caracterização dos biomas estudados neste trabalho, foram utilizados como base um mapa de biomas do Brasil, na escala de 1:5.000.000 (IBGE, 2004a), e um mapa de vegetação do Brasil, na escala de 1:5.000.000 (IBGE, 2004b).

Para avaliação temporal das diferentes fisionomias dos biomas estudados, foi utilizado um conjunto de 144 imagens EVI com resolução espacial de $250 \mathrm{~m}$, período de composição do produto de 16 dias e resolução radiométrica de 16 bits, no período de fevereiro de 2000 a julho de 2006, derivadas do sensor MODIS/Terra, produto MOD13Q1, adquiridas gratuitamente via internet, através do endereço eletrônico: $<$ http://edcimswww. cr.usgs.gov/pub/imswelcome/>.

Para formar uma cena de toda a região do $\mathrm{NEB}$, foram necessários quatro "tiles", que foram mosaicados por meio do software MRT ("Modis Reprojection Tool"), com definição do modelo da terra WGS84. A reamostragem foi realizada utilizando o método do vizinho mais próximo, e o sistema de coordenadas utilizado foi o Latlong.

O programa Convgeotiff (ARAI, 2003), desenvolvido pelo INPE, foi utilizado para realizar

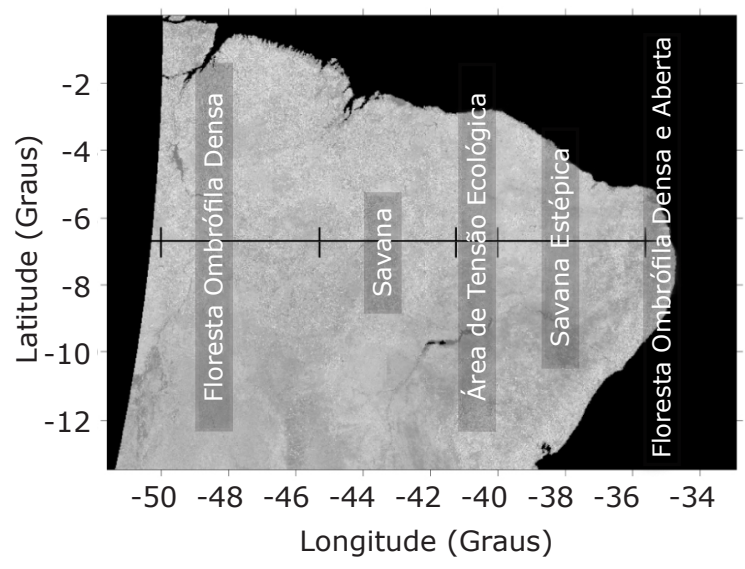

FIGURA 1: Representação do transecto da latitude $-6,69^{\circ}$ na área de estudo.

FIGURE 1: Study area transect at latitude $-6.69^{\circ}$. a conversão das imagens de 16 bits para 8 bits, para serem utilizadas no SPRING (CÂMARA, et al., 1996).

Com a finalidade de diminuir o ruído nos dados originais de EVI, foram aplicados às imagens dois filtros em sequência, um filtro espacial e outro espaço-temporal. O filtro espacial foi aplicado para todas as 144 imagens temporais EVI, sendo do tipo mediana com janela de $7 \times 7$, ou seja, o valor retornado para determinado "pixel" central foi a mediana dos 49 (7x7) níveis digitais dessa janela. Esse filtro tem a capacidade de reduzir os ruídos e de preservar as bordas.

Após a filtragem espacial das imagens, gerou-se uma imagem temporal sob o transecto na latitude $-6,69^{\circ}$, ou seja, para cada imagem, foi capturada a respectiva linha com os dados de EVI nessa latitude, sobrepondo essa linha de dados à linha EVI da data anterior. Dessa forma, formou-se uma imagem temporal para essa latitude, de modo que cada linha dessa imagem representa os dados de EVI de uma determinada data e, as colunas, as diferentes longitudes. A essa imagem temporal foi aplicado outro filtro, também de mediana, porém, com janela de 5x5. A escolha desses filtros foi realizada a partir de diversos testes de tamanhos de janelas e tipos de filtros, sendo estes os que apresentaram melhores resultados.

Para uma análise mais detalhada do comportamento temporal do EVI nos principais biomas, foram selecionadas, dentro do transecto, amostras para representar o comportamento temporal desses biomas, tendo sido escolhidas as seguintes áreas: bioma mata atlântica na longitude $-35^{\circ} 29^{\prime}$, Paraíba; bioma caatinga na longitude -36 $39^{\circ}$, Paraíba; bioma cerrado na longitude $-41^{\circ} 47^{\prime}$, Piauí; e bioma amazônia na longitude -46 $59^{\circ}$ ', Maranhão. Gráficos de EVI temporais desses biomas foram gerados, assim como suas respectivas estatísticas descritivas como, por exemplo, a média $(\overline{\mathrm{X}})$, o desvio padrão $(D P)$ e o coeficiente de variação $(\mathrm{CV})$ pelas fórmulas mostradas abaixo:

$$
\begin{aligned}
& \bar{X}=\sum \frac{E V I_{i}}{n} \\
& D P=\sum \frac{\left(\bar{X}-E V I_{i}\right)^{2}}{n} \\
& C V(\%)=100 \frac{\bar{X}}{D P}
\end{aligned}
$$

em que, $\mathrm{i}=1,2,3 \ldots \mathrm{n}$ sendo $\mathrm{n}$ o número total de imagens EVI (144). 


\section{RESULTADOS E DISCUSSÃO}

Na Figura 2, é apresentado um exemplo do comportamento espacial do EVI do transecto sob latitude $-6,69^{\circ}$ e longitudes de $-36^{\circ}$ a $-37^{\circ}$, referente a fevereiro/2000, dos dados originais e do resultado do EVI após a aplicação do primeiro filtro, mediana de janela $7 \times 7$, que foi aplicado em todas as 144 imagens. Nesse caso, esse transecto está inserido no estado da PB, tendo como bioma predominante a caatinga. Observa-se que o padrão geral do comportamento do EVI ao longo desse transecto dos dados originais foi semelhante ao comportamento dos dados filtrados, com uma redução das variações abruptas dos dados originais.

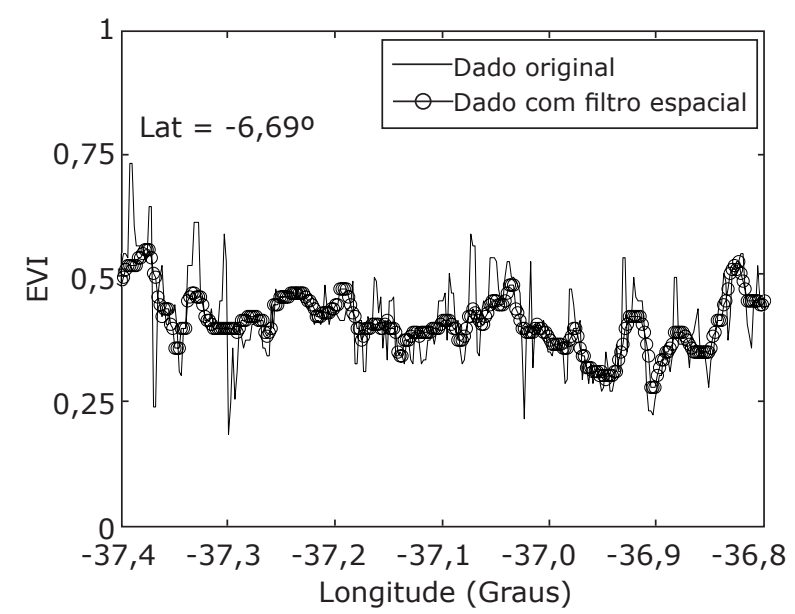

FIGURA 2: Exemplo do processo de filtragem utilizando filtro espacial de mediana $7 \times 7$ nos dados EVI.

FIGURE 2: Example of spatial filter process using median 7 x7 EVI values.

A Figura 3 apresenta a imagem do agrupamento dos transectos sob latitude $-6,69^{\circ}$ de todas as 144 imagens após a filtragem espacial. Nessa figura, o eixo " $y$ " corresponde às datas e o eixo " $x$ " às longitudes. Observa-se, por exemplo, a presença de ruídos nas linhas que apresentam maiores valores de EVI localizadas próximo à data $01 / \mathrm{jul} / 2005$. Esse resultado foi um dos que motivaram a realização da aplicação do filtro mediana de janela $5 \times 5$.

A Figura 4 apresenta a comparação do EVI gerado do primeiro filtro e do EVI do segundo filtro, para a latitude $-6,69^{\circ}$ e longitude $-37,4^{\circ}$, que se refere ao bioma caatinga, apresentando uma variação sazonal característica desse bioma. Observa-se que não houve mudança no comportamento geral da variação temporal do EVI, contudo, os dados abruptos foram suavizados.

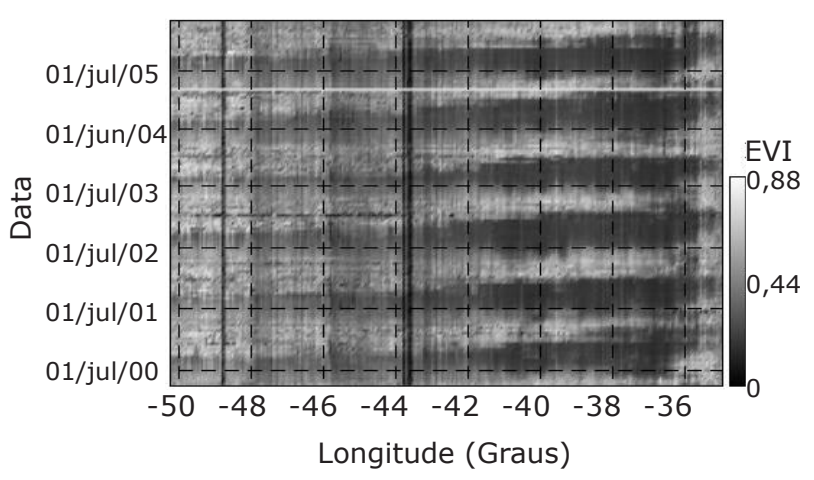

FIGURA 3: Imagem temporal do EVI após o agrupamento das linhas EVI nas diferentes datas no transecto da latitude $-6,69^{\circ}$.

FIGURE 3: Temporal EVI image after clustering EVI lines at transect latitude of $-6.69^{\circ}$ at different periods.

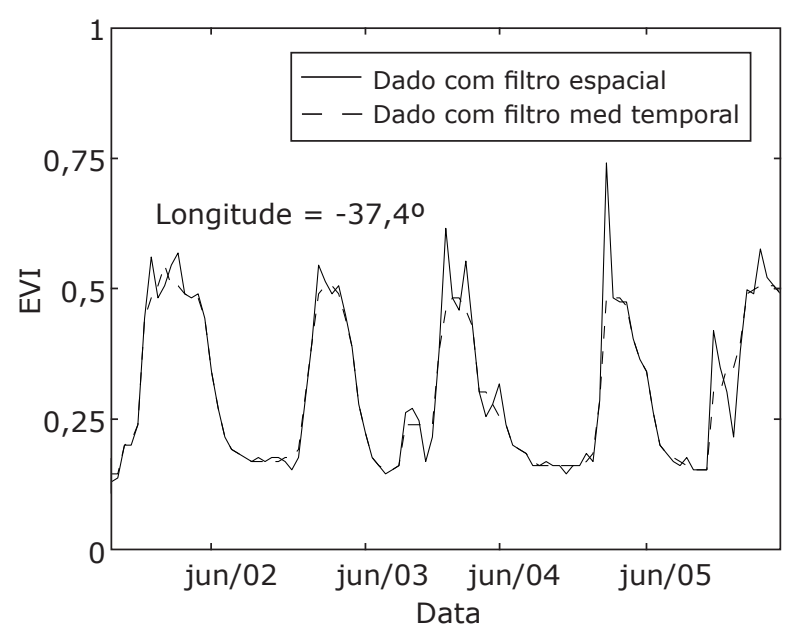

FIGURA 4: Comportamento temporal do EVI na latitude $-6,69^{\circ}$ e longitude $-37,4^{\circ}$ após a segunda filtragem.

FIGURE 4: Temporal behavior of EVI image at latitude -6.69 , longitude -37.4 after applying second filter.

A imagem final resultante da passagem dos dois filtros é mostrada na Figura 5, onde é possível observar uma imagem mais suavizada. As regiões mais claras representam maior quantidade de fitomassa verde, enquanto as regiões mais escuras representam a pouca quantidade ou ausência dessa biomassa. O valor máximo de EVI, após essa última filtragem, foi menor que o anterior, 0,74 contra 0,88 , respectivamente.

O bioma caatinga, por exemplo, (aproximadamente na longitude de $-36^{\circ}$ a $-42^{\circ}$ ), apresenta menores valores de EVI associados a longos períodos de seca. Já no bioma amazônia 


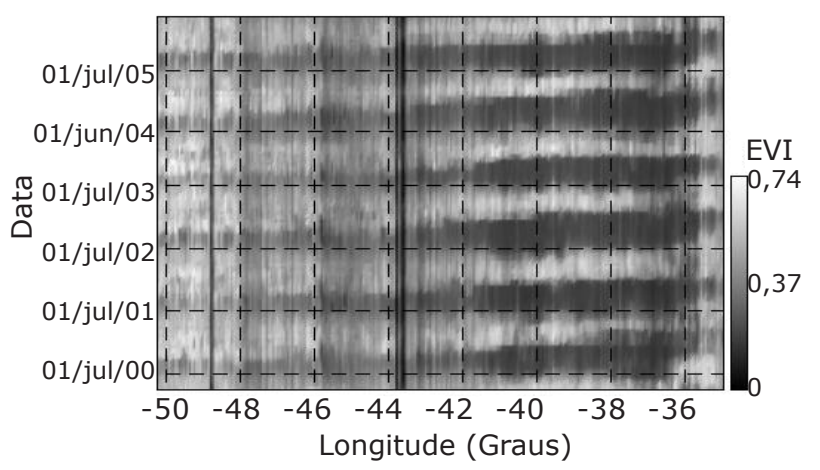

FIGURA 5: Imagem temporal EVI da latitude $-6,69^{\circ}$ após aplicação do segundo filtro (mediana 5x5).

FIGURE 5: Temporal EVI image at latitude $-6.69^{\circ}$ after applying second filter (median $5 \times 5)$.

(aproximadamente na longitude de $48^{\circ}$ a $50^{\circ}$ ), observam-se maiores valores de EVI com ausência de seca prolongada. Nas longitudes aproximadas de -4350', estado do Piauí e -48 50', estado do Tocantins, verifica-se que os valores de EVI são nulos, devido, em ambos os casos, à existência de cursos d'água que passam por essa longitude.

Na Figura 6, são apresentados os diferentes comportamentos de EVI temporal dos biomas aqui estudados. Observa-se que, para todos os biomas, existe um comportamento sazonal típico da fenologia correspondente. Porém, não é correto afirmar que as amostras selecionadas sejam representativas para o comportamento temporal desses biomas, embora sirvam como referência para um possível comportamento típico. A região representada pelo bioma caatinga foi a que apresentou maior variação do EVI ao longo do tempo (coeficiente de variação de 53\%). Já a menor variação foi observada para as regiões representadas pelos biomas amazônia e mata atlântica, ambos com coeficiente de variação de $16 \%$. O bioma cerrado apresentou coeficiente de variação de $22 \%$. Os maiores valores de EVI foram observados para o bioma amazônia, enquanto os menores foram encontrados na região representativa do bioma caatinga.

Quando comparado o EVI temporal dos biomas amazônia e mata atlântica (Figura 6a), observa-se que o bioma mata atlântica apresenta valores de EVI quase tão altos quanto os valores de EVI do bioma amazônia. Observa-se que há uma defasagem dos maiores valores de EVI, o que deve ser causado pela diferença de início e final do período de precipitação característico em cada um dos biomas.
Quanto ao comportamento do EVI temporal dos biomas amazônia e cerrado (Figura 6b), observase uma semelhança na sazonalidade, devido aos períodos de precipitação serem semelhantes, por estarem em regiões geográficas mais próximas, bem como maiores valores de EVI para o bioma amazônia, devido à fenologia de ambos os biomas.

A Figura 6c apresenta comportamento do EVI temporal para os biomas amazônia e caatinga. Observa-se a diferença nos valores de EVI para um mesmo período, resultado da fenologia dos biomas e do regime pluviométrico.

A semelhança do padrão sazonal dos biomas mata atlântica e cerrado é observada através do comportamento temporal do EVI desses biomas, porém, o bioma cerrado apresentou menores valores de EVI (Figura 6d). A defasagem nesses biomas é visível devido ao regime pluviométrico.

O comportamento do EVI temporal nos biomas mata atlântica e caatinga é apresentado na Figura 6e, onde se observa que os valores de EVI para o bioma mata atlântica são, na maioria das vezes, maiores do que os valores de EVI para o bioma caatinga, fato que deve-se à fenologia de ambos os biomas.

A mesma semelhança de sazonalidade pode ser observada na Figura 6f, que apresenta o comportamento do EVI temporal nos biomas cerrado e caatinga.

O comportamento temporal diferenciado entre tipos de vegetação por meio de IVs já foi estudado por outros autores, que também encontraram distinção de comportamento das diferentes fisionomias vegetais (Batista et al., 1993; Lacruz et al., 2005; Liesenberg et al., 2006).

A relação entre EVI e precipitação já foi discutida em alguns trabalhos, como por exemplo, o de Espig et al. (2006), que, utilizando imagens EVI e NDVI em seis áreas da região semiárida do Brasil com existência do bioma caatinga, nos anos de 2003 e 2004, observaram a variação sazonal dessas áreas e constataram que os valores mais elevados de EVI e NDVI ocorreram nos meses de maiores valores de precipitação. Nunes et al. (2007), por sua vez, ao realizarem a comparação da variação sazonal do nível de biomassa para quatro fitofisionomias encontradas no bioma amazônia, estado do Amazonas entre 2004 e 2005, através de dados EVI e NDVI do Sensor MODIS e informações da pluviosidade mensal, verificaram que os menores valores de EVI acompanharam os menores níveis pluviométricos. 

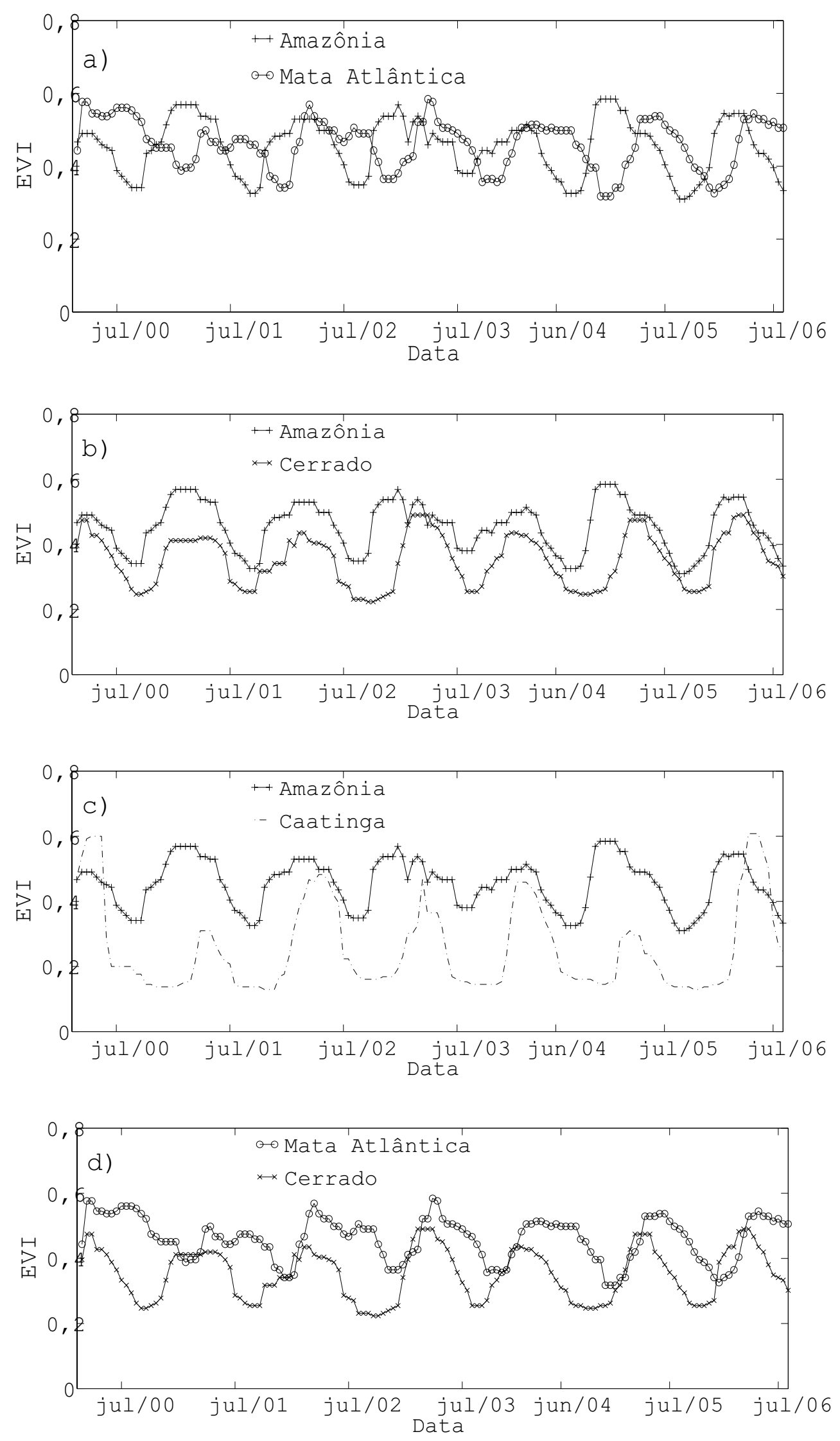

Continua...

Ci. Fl., v. 21, n. 1, jan.-mar., 2011 

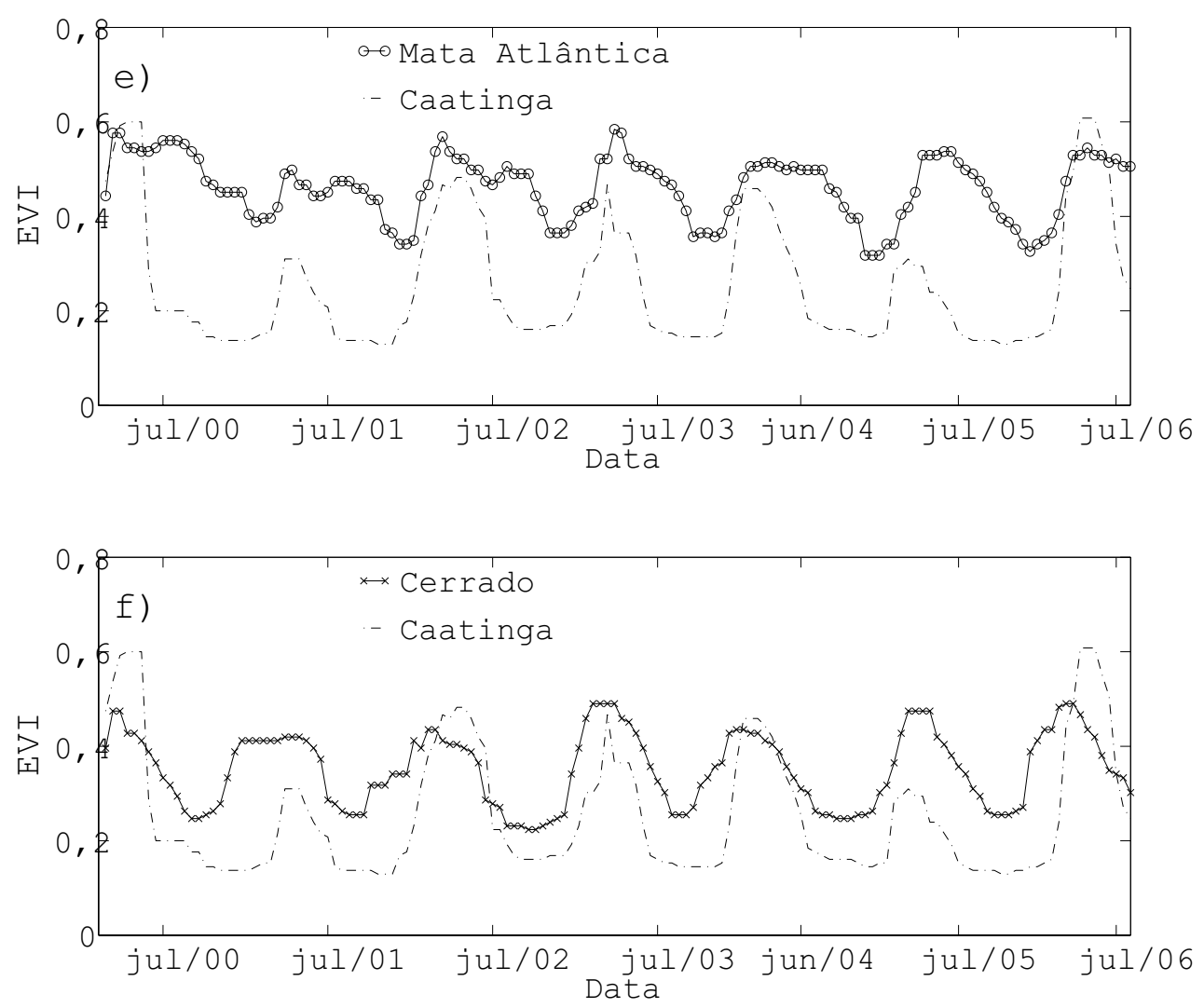

FIGURA 6: Comportamento temporal do EVI na latitude $-6,69^{\circ}$ correspondente aos principais biomas do NEB.

FIGURE 6: Temporal EVI image behavior at latitude $-6.69^{\circ}$ corresponding to the main biome of NEB.

\section{CONCLUSÕES}

Este estudo mostrou a análise do comportamento temporal do EVI do sensor MODIS nos principais biomas do NEB para o período de fevereiro de 2000 a julho de 2006 . A partir dos resultados obtidos foi possível concluir que:

- Para a latitude -6,69\%, o EVI foi capaz de mostrar a diferença sazonal entre os diferentes biomas estudados;

- Dentre os biomas, as regiões representadas pelos biomas amazônia e mata atlântica apresentaram a menor variação sazonal e os maiores valores de EVI;

- O bioma caatinga apresentou maior variação sazonal entre os biomas estudados.

\section{REFERÊNCIAS BIBLIOGRÁFICAS}

ARAI, E. Software ConvGeoTiff. Instituto Nacional de Pesquisas Espaciais, 2003.
BATISTA, G. T.; SHIMABUKURO, Y. E.; LAWRENCE, W. T. Monitoramento da cobertura florestal através de índices de vegetação do NOAAAVHRR. In: SIMPÓSIO BRASILEIRO DE SENSORIAMENTO REMOTO, 7., 1993, Curitiba. Anais... São José dos Campos: INPE, 1993. v. 2, p. 30-37.

CÂMARA, G. et al. Spring: integrating remote sensing and gis by objectoriented Data modeling. Computers \& Graphics, 20: (3) 395-403. 1996. ESPIG, S. A.; SOARES, J. V.; SANTOS, J. R. Variações sazonais do EVI e NDVI em áreas do semi-árido brasileiro. In: SEMINÁRIO EM ATUALIZACÃO EM SENSORIAMENTO REMOTO E SISTEMAS DE INFORMAÇÕES GEOGRÁFICAS APLICADOS À ENGENHARIA FLORESTAL, 7., 2006, Anais... Curitiba: INPE, 2006. p. 219-226. Disponível em: $<$ http://mtc-m12.sid.inpe.br/rep-/sid.inpe.br/ mtc-m12@80/2006/11.07.12.16>. Acesso em: 19 jun. 2007.

FERNANDES, A. Conexões florísticas do Brasil. 
Fortaleza: Banco do Nordeste, 2003. 135 p.

FERREIRA, M. E. et al. Análise da sazonalidade de paisagens antrópicas e nativas do bioma Cerrado através dos produtos MODIS índices de vegetação, área foliar e atividade fotossintética. In: SIMPÓSIO BRASILEIRO DE SENSORIAMENTO REMOTO, 12., 2005, Goiânia. Anais... São José dos Campos: INPE, 2005. p. 505-512. Disponível em: <http:// urlib.net/ltid.inpe.br/sbsr/2004/11.22.17.41>. Acesso em: 22 jun. 2007HUETE, A. R. et al. A comparison of vegetation indexes over a global set of TM images for EOS-MODIS. Remote Sensing of Environment, v. 59, n. 3, p. 440-45, 1997.

IBGE. Mapa de biomas do Brasil. Rio de Janeiro, 2004a.

IBGE. Mapa de vegetação do Brasil. Rio de Janeiro, 2004b.

JUSTICE, C.O.; TOWNSHEND, J.R.G.; HOLBEN, B.N.; TUCKER, C.J. Analysis of the phenology of global vegetation using meteorological satellite data. International Journal of Remote Sensing, v. 6, p. 1271-1318, 1985.

JUSTICE, C.O. et al. The moderate Resolution Imaging Spectroradiometer (MODIS): land remote sensing for global change research. IEEE Transactions on Geoscience and Remote Sensing, v. 36, n. 4, p. 1228-1247, 1998.

LACRUZ, P. M. S.; SANTOS, J. R.; CONFORTE, J. C.Utilização de séries temporais de imagens MODIS/ Terra e análise harmônica para o monitoramento de corredores ecológicos. In: SIMPÓSIO BRASILEIRO DE SENSORIAMENTO REMOTO, 12., 2005, Goiânia. Anais... São José dos Campos: INPE, 2005. p. 3223-3228. Disponível em: <http:// urlib.net/ltid.inpe.br/sbsr/2004/11.22.18.35.39>. Acesso em: 12 out. 2007.

LIESENBERG, V.; PONZONI, F. J.; GALVÃO, L. S. Análise da dinâmica sazonal de índices de vegetação do sensor modis em algumas fitofisionomias do bioma cerrado. In: VII SEMINÁRIO DE ATUALIZAÇÃO EM SENSORIAMENTO REMOTO E SISTEMAS DE INFORMAÇÕES GEOGRÁFICAS APLICADOS À ENGENHARIA FLORESTAL. Curtiba. Anais... 2006. Disponível em: <http://urlib.net/sid.inpe.br/ mtc-m12@80/2006/09.25.13.47>. Acesso em: 30 ago. 2007.

MOREIRA, M. A.; SHIMABUKURO, Y.E. Cálculo do índice de vegetação a partir do sensor AVHRR In: Aplicações ambientais brasileiras dos satélites NOAA e TIROS-N. São Paulo: Oficina de Textos, cap. 4, p. 79-101, 2004.

NUNES, G. M.; SOUZAFILHO, C. R.; FERREIRA, L. G. Monitoramento da cobertura vegetal em áreas de Reservas de Desenvolvimento Sustentável na Amazônia Central através de Índices de Vegetação do Sensor MODIS. In: SIMPÓSIO BRASILEIRO DE SENSORIAMENTO REMOTO, 13., 2007, Florianópolis. Anais... São José dos Campos: INPE, 2007. p. 6893-6899. Disponível em: <http://urlib. net/dpi.inpe.br/sbsr@80/2006/11.15.18.09.34>. Acesso em: 23 nov. 2007.

SILVA, A. F. da. A fitossociologia na Mata Atlântica. IN: SIMPÓSIO SOBRE ECOSSISTEMAS DA COSTA SUL E SUDESTE BRASILEIRA. 1987. São Paulo. Anais... São Paulo: Academia Brasileira de Ciência do Estado de São Paulo, 1987. p. 62-77. TOWNSHEND, J.R.G.; JUSTICE, C.O.; KALB, V. Characterization and classification of South American land cover types using satellite data. International Journal of Remote Sensing, v. 8, p. 1189-1207, 1987.

TUCKER, C.J.; TOWNSHED, J.R.G.; GOFF, T.E. African Land-Cover Classification Using Satellite Data. Science, Washington, v. 227, n. 4685, p. 369375, 1985. 